\title{
The Effect of Cholesterol, Lanosterol, and Ergosterol on Lecithin Bilayer Mechanical Properties at Molecular and Microscopic Dimensions: A Solid-State NMR and Micropipet Study
}

\author{
Emil Endress, ${ }^{\dagger}$ Sybille Bayerl, ${ }^{\dagger}$ Katrin Prechtel $^{\ddagger}{ }^{\prime}$ Christian Maier ${ }^{\ddagger}$ \\ Rudolf Merkel, $\S$ and Thomas M. Bayerl*, ${ }^{*}$ \\ Physikalisches Institut EP5, Universität Würzburg, D-97074 Würzburg, Germany, \\ Physik-Department E22, Technische Universität München, D-85747 Garching, Germany, and \\ Forschungszentrum J ülich, ISG, D-52425 J ülich, Germany
}

Received October 25, 2001. In Final Form: J anuary 21, 2002

\begin{abstract}
Lecithin (DPPC) membranes doped with high concentrations (40 mol \%) of three biologically relevant sterols (cholesterol, lanosterol, and ergosterol) were studied by spectroscopic and force measurements. Micropi pet aspiration measurements of giant unilamellar vesicles provided the membranearea expansion modulus K on the mi croscopic scale while deuterium NM R experiments gave parameters such as the line shape, transverse relaxation, and mol ecular order, which are all based on a mol ecular scal eat a given time resol ution. Micropi pet experiments at $10^{\circ} \mathrm{C}$ gaveK values for thethreesamples with ergosterol $>$ cholesterol $>$ lanosterol. From theNMR weobtained thehighest $\mathrm{CH}_{2}$-segmental molecular order and longest transverse rel axation time for cholesterol and the opposite for ergosterol. The lanosterol NMR parameters were found to be between those of the other two sterols but were cl oser to those of chol esterol. The extent of deformation of the sterol-doped vesicles in the magnetic field of the NMR magnet was lanosterol > cholesterol > ergosterol. The results suggest that membrane micromechanic properties are manifest in solid-stateNMR parameters and suggest that high $\mathrm{K}$ values at the microscopic scale correspond to high molecular order and long transverse relaxation times at the molecular scale. Furthermore, the extent of magnetic field orientation of vesicles measured by NMR correlated well with $\mathrm{K}$ determined by micropi pet aspiration. The results may help to appreciate why cholesterol has gained a dominant role over the other sterols in the course of cellular evolution.
\end{abstract}

\section{Introduction}

Cholesterol, lanosterol, and ergosterol are three structurally similar biological ly relevant sterol swhich dominate the sterol fraction in the mebranes of eukaryotes (cholesterol), of procaryotes (lanosterol), and of certain fungi or protozoans (ergosterol). F rom an evolutionary perspective, lanosterol is the precursor of cholesterol and thelatter did not occur in nature before the Earth's atmosphere turned aerobic. 1,2 It was long speculated that the known variations in the molecular structure (Figure 1) of the three sterols may be in part responsible for their distribution in the different species. But the possible relation between sterol structure and function in the cell membranes is still unclear. Several nuclear magneticresonance (NMR) studies were devoted to the comparison between the sterols in model $^{3-5}$ and natural ${ }^{6}$ membranes. They

* To whom correspondence may be addressed.

† Universität Würzburg.

‡ Technische Universität München.

$\S$ Forschungszentrum J ülich.

(1) Bloch, K. Cholesterol, evolution of structure and function. Biochemistry of lipids and membranes; Benjamin/Cummings: Menlo Park, CA, 1985.

(2) Bloom, M.; Mouritsen, O. G. Biophys. Handb. Membr. 1990.

(3) Brown, M. F.; Thurmond, R. L.; Dodd, S. W.; Otten, D.; Beyer, K. Phys. Rev. E: Stat., Phys., Plasmas, Fluids, Relat. Interdiscip. Top. 2001, 1, 6401.

(4) Urbina, J . A.; Pekerar, S.; Le, H. B.; Patterson, J .; Montez, B.; Oldfield, E. BBA-Biomembranes 1995, 1238, 163-176.

(5) Yeagle, P. L. Biochim. Biophys. Acta 1985, 815, 33-36.

(6) Huang, T. H.; DeSiervo, J. A.; Yang, Q. X. Biophys. J. 1991, 59 $691-702$.

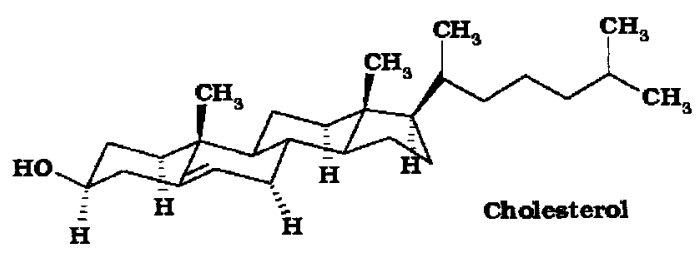

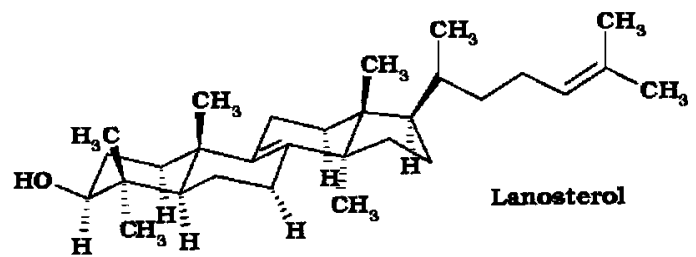

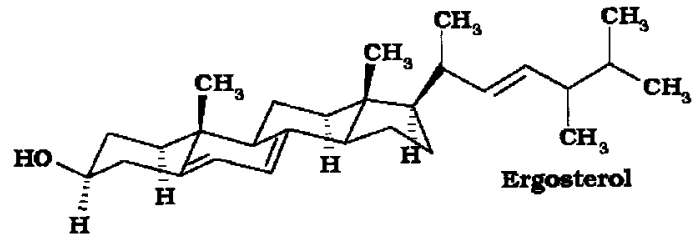

Figure 1. Molecular structures of cholesterol, lanoster ol, and ergosterol.

demonstrated that these sterols show significant differences in their effect on membrane order and NMR relaxation times and suggest that different viscoelastic and morphologic membrane properties may arise from each of these sterols. The influence of cholesterol on the mechanical properties of membranes is well documented. 
Cholesterol substantially increases the area expansion modulus and the yield tension of membranes. ${ }^{7-9} \mathrm{Fur}$ thermore it increases the bending rigidity. However, it appears that the effects of lanosterol and ergosterol on the mechanical properties of membranes have not been studied before.

In this paper, we combined physical methods on the molecular (deuterium NMR) and the microscopic (micropi pet) level for thecomparativestudy of lecithin bilayers doped with high concentrations of the three sterols. The sterol concentration in the bilayer $(40 \mathrm{~mol} \%)$ is in a range where for cholestrol the unique liquid-ordered (lo) phase was observed. Although it is not establ ished yet whether this phase exists also for the other two ster ols (ergosterol and lanosterol ), a comparison at mol ecular and mi croscopic level can provide valuable insight into the mechanisms by which the sterols modulate membrane properties and how molecular properties translate into macroscopic function. Moreover, the combination of the two methods may help to assess how membrane micromechanic properties measured at the microscopic level manifest themselves at the molecular level.

The observables of deuterium solid-state NMR are all dependent on molecular motion in thesampleunder study with respect to a certain time scale. The time scale of NMR spans the millisecond-nanosecond range, and different observables are sensitive to one or several correlation times of the molecular reorientation. In our paper we have studied the observables line shape, order parameter, and transverse relaxation for samples having different membrane micromechanic properties. These membrane properties were determined by a method (micropipet aspiration) which is essentially time scale independent and is rather based on microscopic observation of membraneshapechanges in responsetotheapplied external force, i.e., the suction pressure of the pipet. It is now interesting to explorehow themembranemicroelastic properties determined by a static microscopic technique will show in a dynamical experiment on a molecular scale.

\section{Materials and Methods}

Materials. L- $\alpha$-Dipalmitoyl-phosphatidylcholine (DPPC), its chain perdeuterated analogue (DPPC- $d_{62}$ ), and cholesterol were obtained from Avanti Polar Lipids (Alabaster, AL). Lanosterol and ergosterol were both purchased from Sigma-Aldrich Chemie (Germany). Deuterium depleted water was from Campro Scientific (Germany).

Sample Preparation. For deuterium NMR, DPPC- $d_{62}$ and the sterol were separately dissolved in 65:30:5 chloroform/ methanol $/ \mathrm{H}_{2} \mathrm{O}$ and then volumetrically added to obtain a DPPC$\mathrm{d}_{62} /$ sterol mixture of 6:4 at a DPPC- $\mathrm{d}_{62}$ concentration of $15 \mathrm{mg} /$ $\mathrm{mL}$. The mixtures were lyophilized using liquid nitrogen for 40 $\mathrm{h}$ and then rehydrated using deuterium-depleted water containing $20 \mathrm{mM}$ Hepes buffer at $\mathrm{pH} 7.0$ at $50^{\circ} \mathrm{C}$ for $12 \mathrm{~h}$ under gentle vortexing. After this, the samples were cycled in temperature several times between two water baths of 20 and $50{ }^{\circ} \mathrm{C}$ in order to improve sample homogeneity. Freeze fracture electron microscopy showed that this preparation provided large multilamellar vesicles (MLV) with diameters up to $20 \mu \mathrm{m}$ and thousands of layers (see below).

Oriented samples of the three DPPC- $\mathrm{d}_{62} /$ sterol mixtures were prepared on microscopic glass slides (100 $\mu \mathrm{m}$ thickness) as described in detail in a previous paper ${ }^{10}$ and hydrated via vapor pressure to a water content of 20-22 wt \%. The water content was checked by weighing, using a high-precision scale. Thirty glass slides were stacked and inserted into the cylindrical and

(7) Evans, E.; Needham, D. J . Am. Chem. Soc. 1987, 91, 4219-4228.

(8) Needham, D.; Evans, E. Biochemistry 1988, 27, 8261-8269.

(9) Needham, D.; Nunn, R. S. Biophys. J. 1990, 58, 997-1009.

(10) König, S.; Pfeiffer, W.; Bayerl, T.; Richter, D.; Sackmann, E. J Phys. Fr. 1992, 1589-1615. vapor-tight NMR sample tube. After this, the samples were annealed for $12 \mathrm{~h}$ at $70^{\circ} \mathrm{C}$.

A pure DPPC- $d_{62}$ single bilayer sample on silica beads of 600 $\mathrm{nm}$ diameter was prepared according to procedures described previously. The sample was dissolved in buffered deuterium depleted water and filled into the NMR sample tube $(1 \mathrm{~mL}$ volume) at a concentration of $15 \mathrm{mg}$ of DPPC- $\mathrm{d}_{62} / \mathrm{mL}$.

For micropipet experiments, vesicles were prepared by the electroswelling method. ${ }^{11}$ Mixtures of DPPC with the sterol (mixing ratio 6:4) in organic solution (chloroform) were spread on glass slides coated with indium tin oxide (ITO) and dried under vacuum for $2 \mathrm{~h}$. Two slides coated with the same mixture were arranged at a distance of ca. $0.5 \mathrm{~mm}$ in a chamber filled with $164 \mathrm{mM}$ sucrose solution and subjected to an ac potential of $1 \mathrm{~V}$ and $10 \mathrm{~Hz}$ for $2 \mathrm{~h}$. This procedure gave giant unilamellar vesicles with diameters ranging from 10 to $60 \mu \mathrm{m}$. The vesicles were inspected using an inverted microscope in the interference contrast mode.

Methods. Solid-State Deuterium NMR. ${ }^{2} \mathrm{H}$ NMR measurements have been carried out at 76.7 MHz with a Bruker AMX 500 spectrometer equipped with a Bruker broad-band high-power probe head and a $10 \mathrm{~mm}$ horizontal solenoid sample coil. A quadrupolar echo sequence with a CYCLOPS phase cycling sequence was used. The $90^{\circ}$ pulse length has been determined by the first moment of the spectra to be $6.7 \mu \mathrm{s}$, and the pulse spacing was $15 \mu \mathrm{s}$. The repetition time was $200 \mathrm{~ms}$, and 4096 complex data points with a dwell time of $1 u$ s were collected in quadrature. The temperature was controlled by a Bruker temperature control unit within $\pm 1^{\circ} \mathrm{C}$. The spectra wereobtained by calculating a one-dimensional F ourier transform of the time domain data points starting at the top of the echo.

F reeze F ractureE lectron M icroscopy. Five microliter droplets of the MLV solutions used for NMR were standard frozen in liquid propane, and the samples were fractured and carbonplatinum coated using a Balzers BAF 400 freeze fracture device. The samples were cleaned by floating on a detergent solution and afterward inspected using a Zeiss EM 902 electron microscope.

Micropipet Aspi ration M easurements. Vesicles were observed in a chamber consisting of two parallel glass coverslips, separated by a spacer of about $1 \mathrm{~mm}$ thickness. To al low easy access of the micropipet, the chamber was left open on two sides. Cylindrical glass micropipets with an inner diameter of about $5 \mu \mathrm{m}$ were prepared as described el sewher $e^{8}$ and connected to a mi crometerpositioned water manometer which allowed application of specified suction pressures. A water hydraulic mi cromanipulator (Narishige, Tokyo, J apan) was used for positioning of the pipet. Thechamber was mounted on thestage of an inverted microscope of the Zeiss Axiovert 135TV series (Carl Zeiss, J ena, Germany), equipped with differential interference contrast optics (DIC). Vesid easpiration was observed with a $40 \times$ long distanceobjective (LD Achroplan 40×/0,6 Carl Zeiss). I mages were projected on the chip of a CCD camera (C2400-77, Hamamatsu, Hamamatsu City, J apan) and recorded to videotape. Digitized pictures with a resolution of $164 \mathrm{~nm} /$ pixel were taken at different pressures to determine the geometrical deformations of the vesi des. I mage analysis was done using the program Scion I mage from Scion Corporation. Data treatment was performed as described bel ow.

\section{Results}

1. Deuterium NMR. ${ }^{2} \mathrm{H}$ NMR spectra were obtained both for multilamellar vesicle dispersions (MLV) and for oriented samples of pure DPPC- $d_{62}$ and of mixtures of DPPC- $d_{62}$ with each of the threesterols ( 40 mol \% sterol). We studied the samples at three temperatures: below and above the liquid-gel phasetransition of pure DPPC$\mathrm{d}_{62}$ at 20 and $50{ }^{\circ} \mathrm{C}$, respectively, and at the physiological temperature of $36{ }^{\circ} \mathrm{C}$. The MLV spectra were studied mainly for two reasons. First, we wished to compare the macroscopic magnetic fiel d orientation of the MLV for the three DPPC- $d_{62} /$ ster ol mixtures with that of pure DPPC-

(11) Angelova, M. I.; Soleau, S.; Meleard, P.; Faucon, J .-F .; Bothorel, P. Prog. Colloid Polym. Sci. 1992, 89, 178-182. 


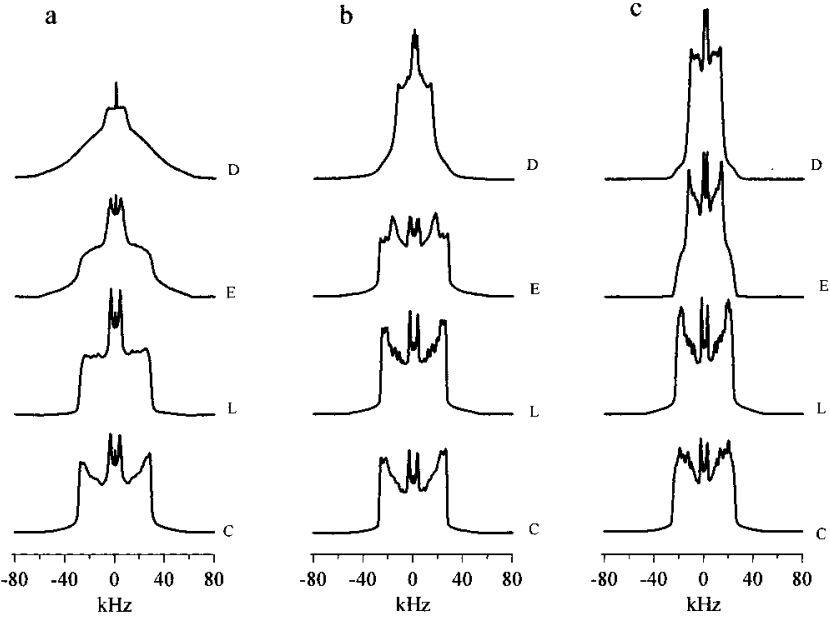

Figure 2. Deuterium NMR spectra of multilamellar dispersions of pure DPPC- $\mathrm{d}_{62}$ and in binary mixture with $40 \mathrm{~mol} \%$ of thesterols, obtained at (a) $20^{\circ} \mathrm{C}$, (b) $36^{\circ} \mathrm{C}$, and (c) $50^{\circ} \mathrm{C}$. Here $D, E, L$, and C denote the spectra of pure DPPC- $d_{62}$, DPPC$\mathrm{d}_{62} /$ ergosterol , DPPC- $\mathrm{d}_{62} /$ lanosterol , and DPPC- $\mathrm{d}_{62} /$ cholesterol, respectively.

$\mathrm{d}_{62}$. Besi de that, the MLVs were used for a comparison of the transverse relaxation time $\mathrm{T}_{2}$ between the samples. Figure 2 shows representative spectra obtained for MLV samples at the three temperatures 20,36 , and $50{ }^{\circ} \mathrm{C}$.

For pure DPPC- $\mathrm{d}_{62}$ we can cl early distinguish between the fluid phase at $50^{\circ} \mathrm{C}$, exhibiting the well-known Pake doublet shape for axially symmetric reorientation of the molecular director axis and with a quadrupolar splitting of $62 \mathrm{kHz}$. I n contrast, thegel phase $\left(20^{\circ} \mathrm{C}\right)$ shows nonaxial symmetric contributions and a much broader line shape as reported previously by Davis. ${ }^{12}$ Finally, the DPPC- $d_{62}$ spectrum at $36{ }^{\circ} \mathrm{C}$ is a superposition of gel and fluid line shape contributions since the actual phase transition temperature of DPPC- $\mathrm{d}_{62}$ is very close at $37^{\circ} \mathrm{C}$.

The presence of the sterols in the DPPC- $d_{62}$ increased for all three samples significantly the quadrupolar splitting and thus the molecular order of the palmitoyl chains. However, it is interesting to note that each of the three sterols modulated the MLV spectral line shape in a different way. This becomes most obvious at $20^{\circ} \mathrm{C}$ where cholesterol exhibits a typical fluid state line shape characteristic for the so-called lo phase ${ }^{13}$ while ergosterol shows much fewer features at the spectral edges and gives the overall impression of a convolution of gel and fluid phasecontributions. Lanosterol at $20^{\circ} \mathrm{C}$ shows lineshape features closer to that of cholesterol but less dominated by the plateau phase of the molecular order parameter distribution, giving the spectrum a more rectangular shape. At the two higher temperatures, the spectra keep distinguishable features for each sterol but generally lanosterol and cholester ol lineshapes appear more closely related than those of ergosterol. In particular, we cannot exclude a slight gel phase contribution to the ergosterol spectrum at $20^{\circ} \mathrm{C}$. In contrast, the spectra at 36 and 50 ${ }^{\circ} \mathrm{C}$ exhibit purely fluid line shapes. Additionally, at $50^{\circ} \mathrm{C}$ a broader feature appears at the spectral shoulders, the origin of which is not clear yet (Figure 2c). The narrower component of this spectrum shows splittings comparable tothose of pureDPPC vesicles. Similar tothelineshapes, the measurement of the transverserelaxation time $T_{2}$ for

(12) Davis, J . H. Biophys. J . 1979, 27, 339-358.

(13) Davis, J . H.; Finegold, L. Themolecular dynamics, orientational order, and thermodynamic phase equilibria of cholesterol/ phosphatidylcholinemixtures: ${ }^{2} \mathrm{H}$ nuclear magnetic resonance; CRC Press: Boca Ratan, FL, 1993.
Table 1. ${ }^{2} \mathrm{H}-\mathrm{NMR} \mathrm{T}_{2}$ Relaxation Times (in $\mu \mathrm{s}$ ), Measured at $36^{\circ} \mathrm{C}$

\begin{tabular}{ccc}
\hline $6: 4$ & $6: 4$ & $6: 4$ \\
DPPC/cholesterol & DPPC/lanosterol & DPPC/ergosterol \\
\hline $586 \pm 10$ & $568 \pm 10$ & $527 \pm 10$
\end{tabular}

Table 2. The Semiaxis Ratio $\epsilon$ Obtained at 36 and $50^{\circ} \mathrm{C}$ for the Three Mixtures and for Pure DPPC-d 62 Coated to a Silica Bead of $640 \mathrm{~nm}$ Diameter as a Control

\begin{tabular}{ccc}
\hline sample & temp, ${ }^{\circ} \mathrm{C}$ & semiaxis ratio $\epsilon^{\text {a }}$ \\
\hline DPPC- $\mathrm{d}_{62}$ & 36 & 1.00 \\
6:4 DPPC/cholesterol & 36 & 0.64 \\
& 50 & 0.63 \\
6:4 DPPC/lanosterol & 36 & 0.60 \\
& 50 & 0.58 \\
6:4 DPPC/ergosterol & 36 & 0.74 \\
& 50 &
\end{tabular}

a Here $\epsilon$ is defined as the ratio of the semiaxis perpendicular to the semiaxis parallel to the static magnetic field $\mathrm{B}_{0}$.

the three sterol samples at $36^{\circ} \mathrm{C}$ showed a dependence on the individual sterol (Table 1). Here cholesterol gave the highest $T_{2}$ value whileergosterol showed theshortest $\mathrm{T}_{2}$.

The MLV spectral line shapes from Figure 2 were analyzed for magnetic field orientation of the vesicles in the field of the NMR magnet, which occurred due to the anisotropy of the diamagnetic susceptibility of the fatty acyl chains of the phospholipids. This caused the fluid vesicles to adopt an ellipsoidal shape with the long axis parallel to the magnetic field direction $\mathrm{B}_{0},{ }^{14}$ resulting in characteristic line shape distortions. Bending energy of the bilayers is one of the major forces to compensate the deformation toward ellipsoidal shapes. As a result, vesides in the gel phase where the bilayer is virtually frozen and largel y inelastic do not show magneticorientation effects. However, in the fluid phase the extent of deformation may serve as a crude measure for the microelastic properties of thebilayer, in particular its bending energy.

The line shape analysis was performed by fitting the convolution of oriented spectra with ellipsoidal lineshapes to the MLV spectra with the aspect ratio $\epsilon$ (short axis/long axis, i.e., thelower the value of $\epsilon$, the higher the magnetic field orientation) as a parameter according to the line shape function

$$
\mathrm{p}(\theta) \frac{\partial \theta}{\partial \omega}=-\frac{1}{2} \epsilon^{3} \frac{2 \mathrm{x}-2 \omega+\epsilon^{2} \mathrm{x}+2 \epsilon^{2} \omega}{3 \mathrm{x}(3 \mathrm{x}(\mathrm{x}+2 \omega))^{1 / 2}}
$$

derived by Pott et al. ${ }^{15} \mathrm{Herep}(\theta)$ is the probability function of finding the normal of a surface el ement oriented at an angle $\theta$ with respect to the magnetic field direction, $\epsilon$ is theaspect ratio (for $\epsilon=1$ results thewell-known spherical line shape function $\left.(3 x(x+2 \omega))^{-1 / 2}\right), \omega$ is the frequency of the MLV data, and $x$ is the frequency of the oriented data. As can be inferred from Table 2, the MLV sample containing ergosterol $(\epsilon=0.74)$ showed the lowest magnetic field orientation at $36^{\circ} \mathrm{C}$, while it was highest for lanosterol $(\epsilon=0.60)$. The cholesterol MLV exhibited a slightly higher resistance toward magnetic fiel d orientation $(\epsilon=0.64)$ as compared to lanosterol. At $50{ }^{\circ} \mathrm{C}$ the magnetic field orientation for lanosterol is still slightly higher than that for cholesterol. The corresponding value for ergosterol is not given in Table 2 due to the complex (apparently superimposed) line shape of the spectrum.

(14) Brumm, T.; Mops, A.; Dolainsky, C.; Bruckner, S.; Bayerl, T. M. Biophys. J . 1992, 61, 1018-1024.

(15) Pott, T.; Dufourc, E. J . Biophys. J . 1995, 68, 965-977. 


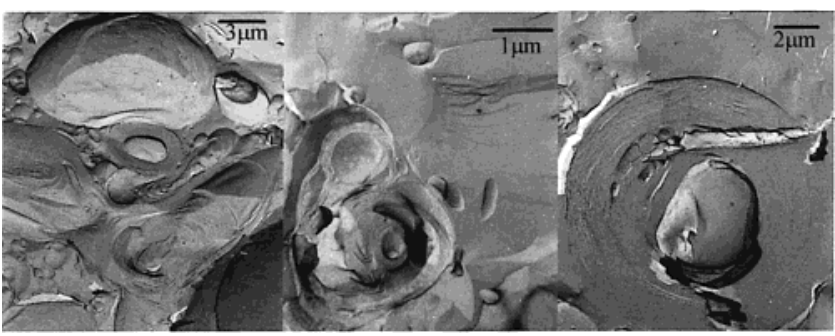

Figure 3. F reezefractureelectron mi crographs of the samples studied by NMR. Representative pictures are shown for DPPC$d_{62} /$ cholesterol (left), DPPC- $d_{62} /$ lanosterol (middle), and DPPC$d_{62} /$ ergosterol (right). The bars represent $1 \mu \mathrm{m}$.

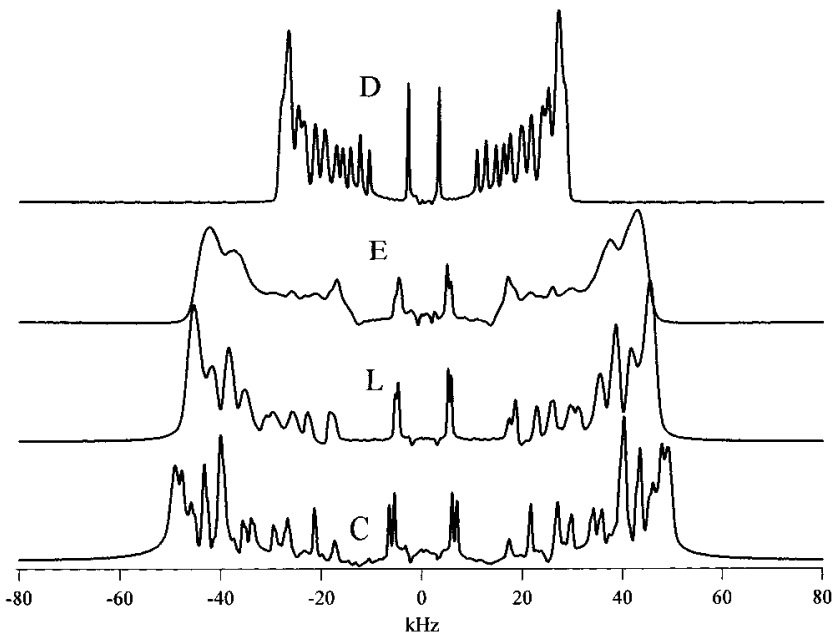

Figure 4. Oriented deuterium NMR spectra of the binary mixtures of DPPC- $d_{62}$ with $40 \mathrm{~mol} \%$ sterol and of pure DPPC$\mathrm{d}_{62}$ obtained at $50^{\circ} \mathrm{C}$. Here $\mathrm{D}, \mathrm{E}, \mathrm{L}$, and C denote the spectra of pure DPPC- $d_{62}$, DPPC- $d_{62} /$ ergosterol, DPPC- $d_{62} /$ lanosterol, and DPPC- $d_{62} /$ chol esterol, respectively.

Theerror of the determination was less than $5 \%$ as verified by applying the fitting procedure to the spectrum of a pureDPPC- $\mathrm{d}_{62}$ bilayer supported by spherical silica beads which undergo no field orientation at all. ${ }^{14}$

To explore the possibility that differences in the MLV morphol ogy for thethreesterol s account for the variation in the value of $\epsilon$, the samples were studied by freeze fracture electron microscopy. All three samples showed highly multilamellar structures with diameters in the range of $0.5-10 \mu \mathrm{m}$ (F igure 3). N o significant differences in size and multilamellarity were observed between cholesterol and lanosterol samples. However, ergosterol showed average MLV sizes moreat the upper end $(10 \mu \mathrm{m})$ of the size range and in some cases the MLV were even larger. Additionally, this sample showed a small population of bi- or trilamellar vesicles with diameters of 200$400 \mathrm{~nm}$. However, their proportion was too low to contribute more than $1-2 \%$ of the total NMR signal.

Sincethelineshape distortions dueto MLV orientation effects may interfere with the determination of the molecular order parameter profile of theDPPC- $d_{62}$ chains using the de-Paking method, ${ }^{16}$ we measured oriented samples of the three mixtures at 36 and $50{ }^{\circ} \mathrm{C}$. Typical spectra obtained are shown in Figure 4, and the order parameter profiles obtained from these spectra $\left(\mathrm{S}_{\mathrm{CD}}\right.$ VS carbon position along the palmitoyl chain) are given in Figure 5.

Although the profiles exhibited a similar shape for all three sterols, there are significant differences regarding

(16) Schäfer, H.; Mädler, B.; Sternin, E. Biophys. J . 1998, 74, 1007-
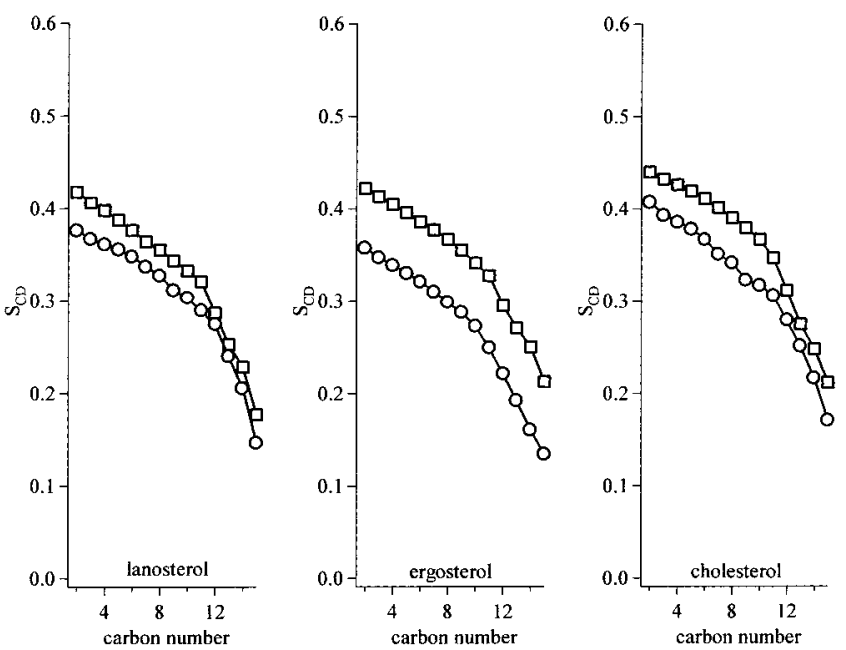

Figure 5. Order parameter profiles obtained from theoriented spectra at $36{ }^{\circ} \mathrm{C}$ (squares) and $50{ }^{\circ} \mathrm{C}$ (circles).

their temperature dependence. The ergosterol profile clearly showed thehighest temperaturedependencewhile the lanosterol profile was significantly less temperature dependent. At $36{ }^{\circ} \mathrm{C}$ the values of $\mathrm{S}_{\mathrm{CD}}$ were very similar for all three samples while at $50{ }^{\circ} \mathrm{C}$ the DPPC chains showed lowest order in the presence of ergosterol and highest $\mathrm{S}_{\mathrm{CD}}$ for cholesterol.

2. Micropi pet Measurements. Micropipet aspiration experiments were performed in order to correlate micromechanic properties of the three sterol-doped DPPC bilayer (same sterol content as for the NMR experiment, i.e., 40 mol \%) samples with the NMR results. In these experiments, we determined the area expansion modulus by the method developed by Evans and co-workers. ${ }^{17}$ Figure 6 represents a typical experimental setup where a giant unilamellar vesicle (GUV) of radius $r_{0}$ was aspirated to the micropipet (radius $r_{p}$ ). It is noteworthy that also in this preparation ergosterol appeared to increasevesidesize. Duetothesuction pressure, p, applied to the vesicle, the excess membrane area (usually less than $5 \%$ of the total area)

$$
\Delta \mathrm{A}=2 \pi \mathrm{r}_{\mathrm{p}}\left(1-\frac{\mathrm{r}_{\mathrm{p}}}{\mathrm{r}_{0}}\right) \Delta \mathrm{L}
$$

was sucked into the pipet up to the projection length $\Delta \mathrm{L}$ with respect to the projection length at vanishing aspiration pressure. Due to the suction, the bilayer surface experiences an isotropic surface tension

$$
\tau=\frac{\mathrm{pr}_{\mathrm{p}}}{2-2 \mathrm{r}_{\mathrm{p}} / \mathrm{r}_{0}}
$$

The change of area with tension is a measure for the deformation elasticity and is given by the compressibility modulus

$$
\mathrm{K}=\mathrm{d} \tau / \mathrm{d} \alpha
$$

with $\alpha$ being the relative change in area, $\Delta \mathrm{A} / \mathrm{A}_{0}$. Plots of $\tau$ Vs $\alpha$ gave straight lines, theslope of which gave thearea compressibility modulus K (Figure 7). The data were independent of the history of the vesicle, i.e., increasing and decreasing the aspiration pressure yielded identical results. This observation excludes artifacts due to area loss or leaky membranes. The values of $\mathrm{K}$ are represented

(17) Evans, E.; Kwok, R. Biochemistry 1982, 21, 4874-4879. 


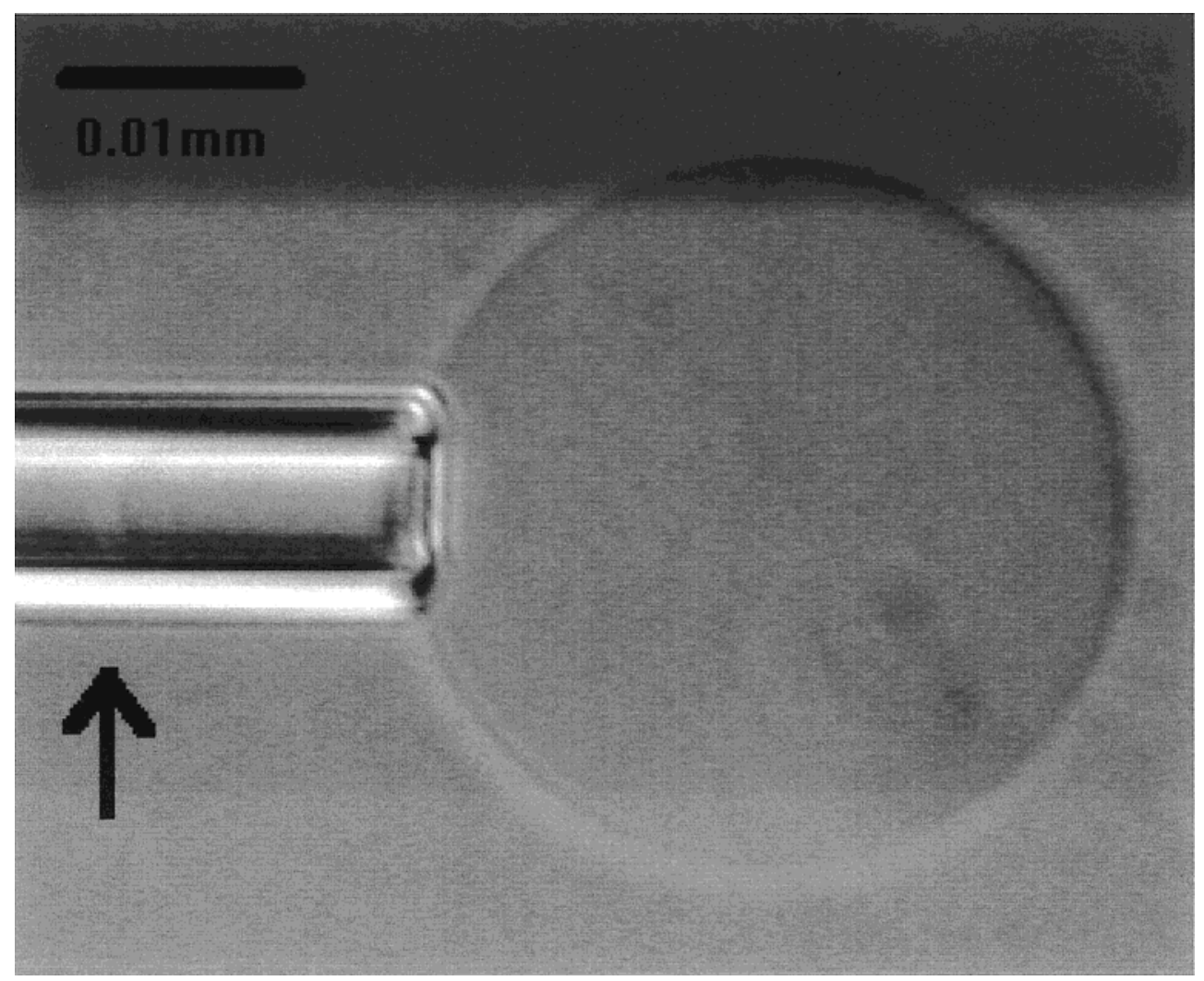

Figure 6. Representative picture of a giant lipid bilayer vesicle (here 6:4 DPPC/ergosterol) with $32 \mu \mathrm{m}$ diameter aspirated to a micropipet. The projection length of the excess membrane area can easily be detected by the change of gray level in the micropipet (arrow).

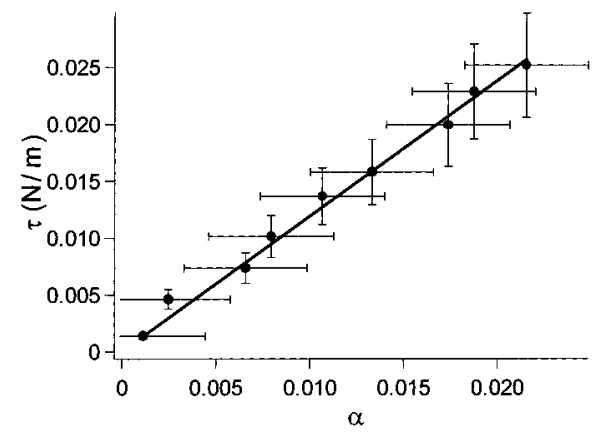

Figure 7. Typical plot of the membrane tension $\tau$ vs area dilatation $\alpha$. The slope of $\tau$ vs $\alpha$ corresponds to the area compressibility modulus $\mathrm{K}$.

Table 3. Area Compressibility Moduli $\mathrm{K}$ (in $\mathrm{mN} / \mathrm{m})$ and the Lysis Tension $\tau_{\mathrm{c}}$ (in $\mathrm{mN} / \mathrm{m}$ ), Obtained for Giant Unilamellar Vesicles by the Micropipet Aspiration Method

\begin{tabular}{ccc}
\hline $\begin{array}{c}\text { lipid-sterol } \\
\text { mixture }\end{array}$ & $\begin{array}{c}\text { area compressibility } \\
\text { modulus, } \mathrm{K}(\mathrm{mN} / \mathrm{m})\end{array}$ & $\begin{array}{c}\text { Iysis } \\
\text { tension }\end{array}$ \\
\hline 6:4 DPPC/cholesterol & $740 \pm 100$ & $23 \pm 3$ \\
6:4 DPPC/lanosterol & $610 \pm 90$ & $23 \pm 5$ \\
6:4 DPPC/ergosterol & $1100 \pm 100$ & $25 \pm 9$
\end{tabular}

along with the critical Iysis tension $\tau_{\mathrm{c}}$ in Table 3 . At a certain critical tension $\tau_{c}$ thevesicles collapse. Weobtained similar values for all three sterol/DPPC GUV samples. However, K varied by nearly a factor of 2 with lanosterol giving thelowest $K$ and ergosterol the highest one. Results for cholesterol-containing samples agree well with literature values. ${ }^{8}$ The variation in $\mathrm{K}$ correlated with the GUV size, where the ergosterol sample showed a larger diameter than that of lanosterol GUV. Similar to the case of the $\epsilon$ values determined by NMR (Table 2), cholesterol gave $\mathrm{K}$ values between those obtained for the other two sterols but closer to lanosterol (Table 3).

\section{Discussion}

Themost interesting result of our study is thecorrelation between micromechanic membrane properties measured by a (static) method at the microscopic scale with parameters derived from a time-resolving method (NMR) which is sensitive at molecular scale. We observed a correlation between the N MR transverse relaxation time $\mathrm{T}_{2}$ (Table 1), the NMR line shape (expressed by the aspect ratio $\epsilon$, Table 2), and the area compressibility modulus $\mathrm{K}$ (Table3) for the three DPPC/sterol samples studied. This correl ation seems quite understandablefrom the physical point of view. Theshortest $\mathrm{T}_{2}$ was measured for ergosterol, indicative for the most rigid (solidlike) bilayer, and its $\epsilon$-value indicated the lowest deformation in a magnetic field and it gavethehighest K. On theother side, lanosterol showed medium $\mathrm{T}_{2}$ (in agreement with a previous study

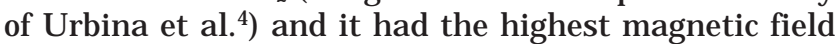
deformation and the lowest $\mathrm{K}$. Obviously the micromechanical properties of membranes must bemost profoundly influenced by the molecular packing of its constituents. Therefore the observed correlation between the results of deuterium NMR line shape and relaxation, which is sensitiveto molecular packing, and micropi pet aspiration, testing the elastic properties of membranes, was no surprise. As a consequence, this finding implies that deuterium NMR may be used to study aspects of membrane micromechanic properties. An interesting question is why theorder parameter profiles measured did not show such a clear correlation (Figure 5). After all, $\mathrm{S}_{\mathrm{CD}}$ is highly 


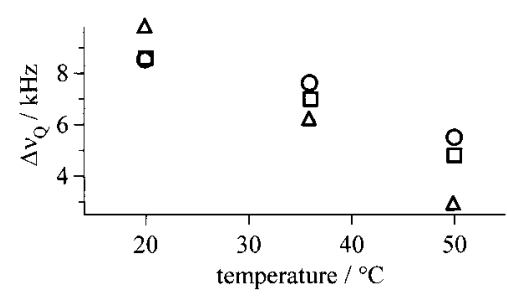

Figure 8. Methyl peak splittings versus temperatureextracted from multilamellar vesicle spectra of 6:4 DPPC-d $\mathrm{d}_{62} /$ ergosterol (triangles), 6:4 DPPC-d 62 /lanosterol (squares), and 6:4 DPPC$\mathrm{d}_{62} /$ cholesterol (circles) as a function of temperature.

sensitive to the molecular area accessible to the chain segments ${ }^{18}$ and the other results strongly suggest that a different degree of li pid area condensation duetothesterol presence is crucial for modulating the membrane micromechanic properties. It should also be noted that in a paper of Urbina et al. ${ }^{4}$ the overall order in DMPC/sterol mixtures (30 mol \% sterol) of MLV was reported as ergosterol $>$ cholesterol $>$ lanosterol. To resolve this discrepancy, we haveto consider thetemperatures where the $S_{C D}$ measurements were performed. Owing to the possible contribution of gel phase to the ergosterol spectrum at $20^{\circ} \mathrm{C}$, we have chosen to anal yze the order parameters for the spectra obtained at 36 and $50^{\circ} \mathrm{C}$ only. However, Figure 5 shows clearly that the temperature dependence of the methylene $S_{C D}$ is quite different for the three samples with the ergosterol sample showing the highest sensitivity to temperature changes. Since the micropipet measurements were performed at low temperature, we would need to extrapolate $S_{C D}$ to this temperatureto compare correctly. H owever, this approach may introduce severe errors owing to possible nonaxial symmetriccontributionstothespectra. Therefore, wehave studied theorder parameter of theterminal methyl groups which are not affected in their axially symmetric reorientation down to temperatures far bel ow $0{ }^{\circ} \mathrm{C} \cdot{ }^{12,19} \mathrm{~F}$ igure 8 shows $S_{C D}$ of the terminal $C_{3}$ group for the three samples as a function of temperature. It demonstrates that at $20{ }^{\circ} \mathrm{C}$, the ergosterol sample shows indeed the highest order while this changes with increasing temperature to lowest order of all three samples at $50^{\circ} \mathrm{C}$. A rationale for this significantly higher temperature sensitivity of the ergosterol sample is not given here; we can only surmise that some uniaxial rotation of this sterol might becomeactivated in this temperaturerange, thereby increasing the separation between adjacent DPPC molecules.

However, the terminal methyl group order parameter at low temperature suggests that indeed high molecular order seems to correspond to the high area compressibility modulus $\mathrm{K}$ obtained for the ergosterol sample. At low temperature $\left(20^{\circ} \mathrm{C}\right)$, our results are also in agreement with those of Urbina et al. 4

It is interesting to discuss our results in terms of the differences in the molecular sterol structure (Figure 1). It appears quite conceivable that cholesterol with its flat sterol body at oneside can achi evea higher packing in the DPPC bilayer and consequently force the acyl chains to adopt a higher order compared to lanosterol. The latter exhibits due to its additional three methyl groups a (in the cross section) bulkier sterol body. These differences in packing along with the resulting different dispersion

(18) Gawrisch, K.; Holte, L. L. Chem. Phys. Lipids 1996, 81, 105116

(19) Hetzer, M.; Gutberlet, T.; Brown, M. F .; Camps, X.; Vostrowsky, O.; Schönberger, H.; Hirsch, A.; Bayerl, T. M. J . Phys. Chem. A 1999 103, 637-642. forces in the bilayer plane may account for the higher values of $\mathrm{K}$ and $\epsilon$ observed for cholesterol over lanosterol. It is less obvious which molecular features of ergosterol enablethis sterol to causean even higher molecular order than cholesterol, along with the highest values for $\mathrm{K}, \epsilon$, and vesi clesize. I ts sterol body exhibits a similar flatness as cholesterol, so weassume that the modifications of the short alkyl chain may account for an even stronger van der Waals interaction between the palmitoyl chains of the DPPC (possibly at the expense of membranefluidity) and thus cause this high membrane bending stiffness. A conceivable sensitive mechanism might be a slightly different average position of theergosterol center-off-mass in the membrane with respect to the bilayer normal direction due to the modifications in the acyl chain as compared to cholesterol.

An important question regarding our comparison of the modulation of DPPC membrane properties by the three sterols studied is whether the lo phase reported for cholesterol is also a feature of the other two sterols. Unfortunately, no published work is available on DPPC/ lanosterol or DPPC/ergosterol phase diagrams. H owever, our NMR results obtained at $20{ }^{\circ} \mathrm{C}$ (Figure 2 ) indicate that lanosterol shows a similar axially symmetric spectrum as cholesterol and also an increase of the DPPC- $d_{62}$ quadrupolar splittings. This indicates that at least the fluid/gel phasetransition of DPPC- $d_{62}$ at $36^{\circ} \mathrm{C}$ is abolished by the presence of lanosterol in a similar way as was observed for cholesterol. Indeed, a microcalorimetry (differential scanning calorimetry, DSC) measurement of the DPPC/lanosterol sample did not show any remaining phase transition. Also for DPPC/ergosterol the DSC gave no detectablethermal event. However, theNMR spectrum of DPPC/ergosterol at $20{ }^{\circ} \mathrm{C}$ (Figure 2) may indicate contributions of gel phase, but at $36{ }^{\circ} \mathrm{C}$ it still appears largely axially symmetric and thus indicative for a fluid phase. All three different types of membranes behaved like fluid membranes in the micropipet experiments, i.e., membranes that were not tensed exhi bi ted largethermal fluctuations. Remaining plastic deformations were not observed following aspiration and expulsion of vesicles. Thus the membranes are fluid on a micrometer length scale. From these observations we deduce that all three sterols render the DPPC to maintain its fluid state bel ow the DPPC phase transition temperature but force the palmitoyl chains toadopt a significantly higher molecular order. F urther studies will berequired toverify or dismiss theexistence of a lo phasefor theergosterol and lanosterol samples.

I t is very tempting to view the results of our study from the perspective of cellular evolution. All three sterols are found in cells, most prominently cholesterol in eukaryotes, but lanosterol exists in prokaryotes while ergosterol is essential in somefungi or protozoan cells. F rom theresults of our study we can speculate that the proven ability of thethreesterolstomodulatethemicromechanic properties of lecithin bilayers to a different degree may have functional reasons and originates from an evolutionary optimization process However, further studies of mixtures with different lipids and even proteins at various amounts of sterols will be required to substantiate our surmise. Neverthel ess, chol esterol seems to bethe perfect mol ecule to keep a del icate balance between membrane rigidity to al low for large cell volumes as is the case for eukaryotes and membrane softness and fluidity to enable complex membrane function. 


\section{Conclusion}

The comparison of the membrane effects of the three sterols suggests at least two molecular mechanisms by which these molecules may modulate membrane properties. One likely candidate is the average depth of immersion of thester ol moleculewith respect tothehydrophobic region of the bilayer. The second may be the extend to which the sterols are able to perform fast uniaxial reorientation with respect to the bilayer normal and the thermal energy barrier at which this rotation is activated.
A quasi-elasticneutron scattering study of thethreeDPPC/ sterol samples to be published elsewhere will provide the mol ecular details and correlation times of these motions.

Acknowledgment. We acknowledge Professor Michael F. Brown and Professor Klaus Müller for valuable discussions. This work was supported by grants from the Deutsche Forschungsgesellschaft (Ba1125/5 and SFB266D13).

LA011596M 